\title{
Defining and describing human-powered products: exploring diverse applications of future technology
}

\author{
Paul Johnson 1, a, Hyunjae Shin ${ }^{2, b}$ and Dr. Luke Harmer ${ }^{3, c}$ \\ ${ }^{123}$ Nottingham Trent University, Burton St., Nottingham, NG1 4BU, UK \\ apaul.johnson@ntu.ac.uk, ${ }^{b}$ daniel.shin@ntu.ac.uk, 9 luke.harmer@ntu.ac.uk
}

Keywords: Human-powered product, product design

\begin{abstract}
The research has monitored both real time and concepts of human-powered products (HPP) ranging from conscious user interaction and fun concepts, to parasitic harvesting concepts. These 'products' have been characterised and mapped onto an 'Interaction Map' which is defined and described by two intersecting dimensions: one is defined by a sub/conscious user interaction and the other is defined by the mechanism of the product. This paper presents the results of a case study conducted with first year product design undergraduate students at Nottingham Trent University in January 2011. Students were briefed to select an electronic product and (re)design it into an interactive 'off the grid product', where its functional power is not being supplied by neither the power grid nor any kind of technology driven power units such as photovoltaic power cells. The results produced a comparative analysis, mapping student project concepts against results from real time HPP monitoring of existing products. Many HPP concepts arose from this study, and the design approach highlighted potential applications of human-power systems, more specific form of engineering requirements, as well as insight into further potential future technological approaches for HPP.
\end{abstract}

\section{Introduction}

The scope of current research is to investigate how a practice based approach of designing new interactivity in HPP - as a means of de-routinizing the current habitual energy consumption through new interactions - can harness and promote sustainable energy consumption in everyday life. The research creates a persuasive argument that there are other notions to consider in wider design contexts for greater benefits beyond the current norm - an unsustainable, over-reliance on technology from traditional power sources which contextualizes ways in which products are designed, and therefore consumed.

The challenge for harnessing human power is finding ways to match various systems with the energy needs of any given device or product. Therefore, the research has been monitoring both tangible (real) products, and concepts of HPP - especially studying power generation methods and mechanisms - ranging from using conscious user interaction concepts, fun aspects through to parasitic harvesting concepts. These 'products' have been characterised and mapped onto an 'Interaction Map' which is defined and described by two intersecting dimensions: one is defined by a sub/conscious user interaction and the other is defined by the mechanism of the product. The attributes of HPP identified in this map will be used as tool to forecast the potential design spaces, and future scenarios/ideas of human-powered products.

\section{Interaction Map}

The method of interaction embedded in HPP communicates directly to users an environmental benefit of harvesting your own energy as alternative power source - a clean energy. One such interactivity, power generation, in HPP may be interpreted as educational intervention, i.e. being told what to do, rather than parasitic, but will always inform and communicate to people what our body can do.

From monitoring concepts of HPP from wider sources, this research developed an 'Interaction Map', shown in Fig. 1. This map is configured around two dimensional axes; the horizontal axis 
represents the user interaction of conscious and sub-conscious power generating interaction of HPP; the vertical axis of this map broadens out the definition of HPP compared to other related literatures where they are all defined as devices powered from an electrical storage unit. The purpose of this map is to characterise and analyse different attributes of HPP.

Examples of such differentiation can be observed from Figs. 2 and 3. The BayGen Radio (left) requires a one hand cranking physical motion to wind up the spring which then unwinds to power the motor to supply sufficient electricity [1]. In this design application and interaction, the users utilise their muscle power to apply a kinetic energy consciously by cranking.

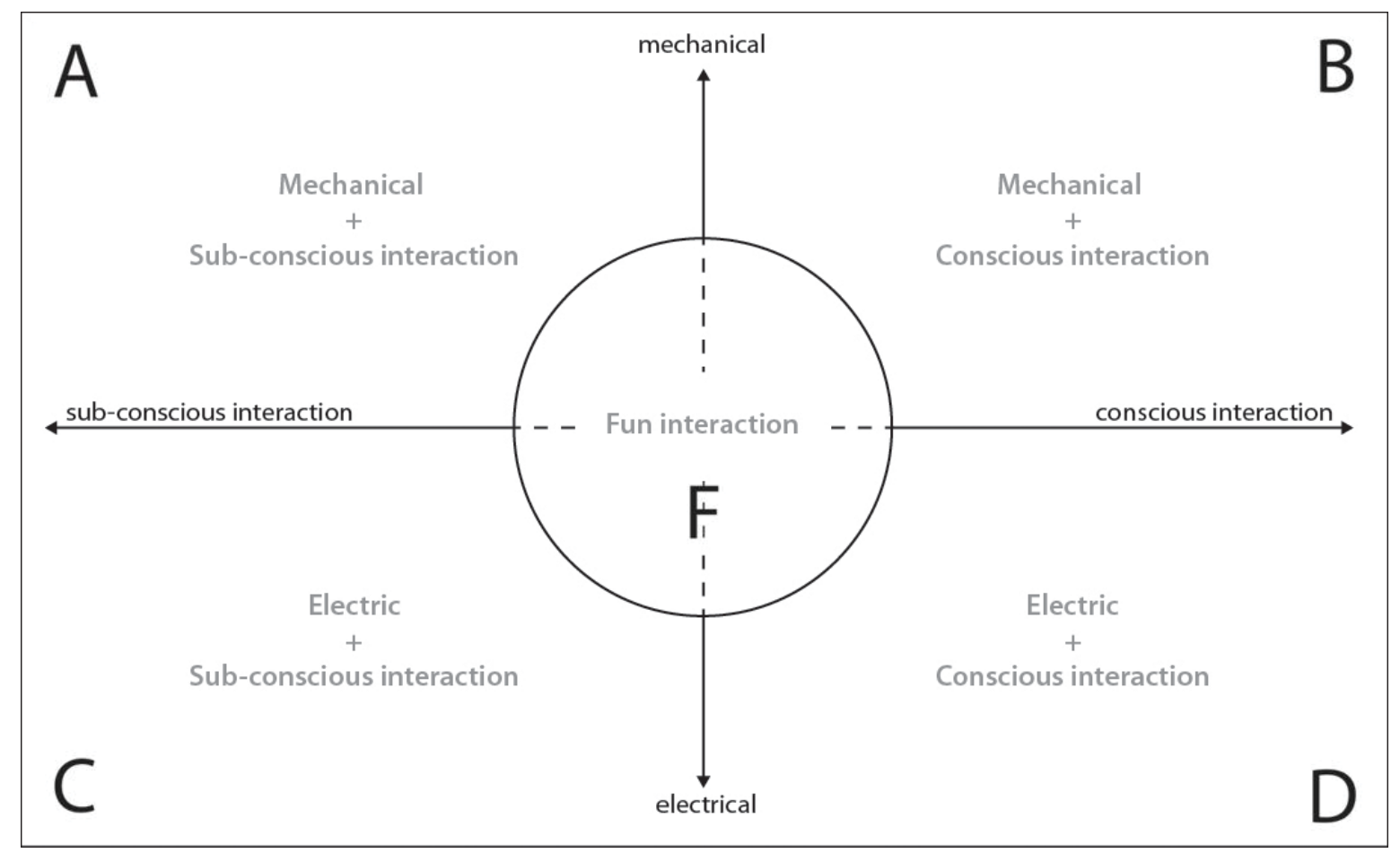

Fig. 1. Interaction Map

For a sub-conscious user interaction, a strong example is the piezoelectric system implemented by East Japan Railway Company (refer to Fig. 3.). The company installed a piezoelectric floor in subway stations where the system will harvest a crowd's footstep energy (footfall) into electricity to power ticket gates and display systems. In this application, the design harvests energy in an ancillary way whilst people are sub-consciously applying their walking energy.

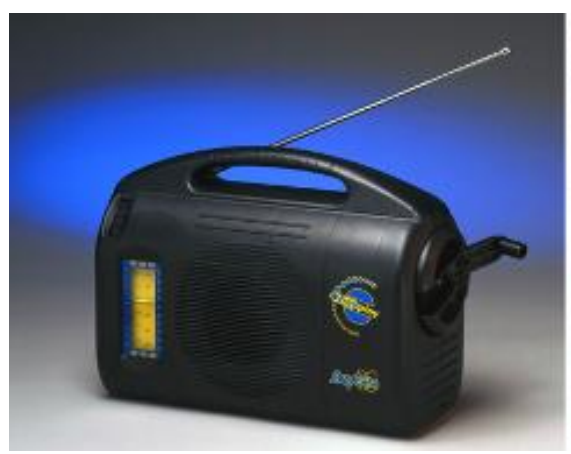

Fig. 2. BayGen Radio [1]

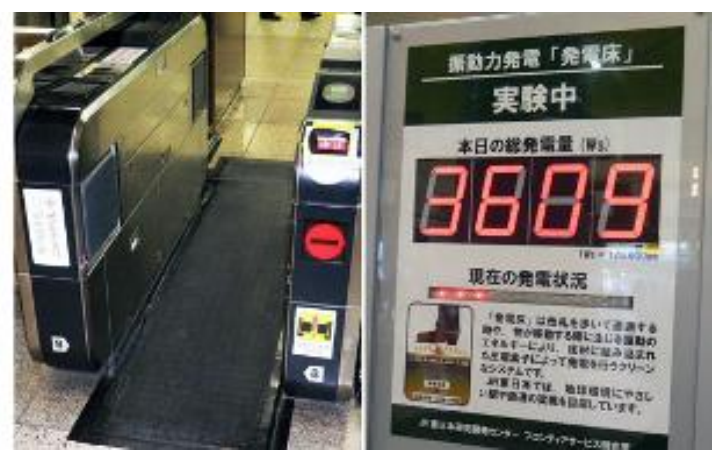

Fig. 3. Piezoelectric system in Tokyo Subways [2]

The leading scholar in the field of HPP is Arjen Jansen. His definition is a 'non-conventional power source' focusing on products featuring a conversion of muscular work into electricity [3]. However, in this research, the HPP is defined as any products powered by human physical interaction 
which can be either mechanically or electrically powered. The best example to explain the vertical axis of the map is the bicycle. It amplifies human muscle energy into mechanical work that produces the velocity of a product which then enables the bicyclist to travel a distance. A bicycle is likely to be mapped within the top zone of the map due to it being a product mechanically powered by humans but not incorporating any electrical storage.

In addition to these two axes, a 'fun' application and range has been inserted in the central zone of the map. An example of this category is the Soccket, shown in Fig. 4. It is a soccer ball that captures the energy during game play to charge batteries. After playing, the user can return home and use the ball to connect a LED lamp to read, study, or illuminate the home [4]. The 'fun' feature, playing a soccer game, is encouraging users to create kinetic energy from a physical interaction. Any 'labour' in terms of energy generation in this device is compensated by an increase in other ways. From this description, the Soccket will be located in the central 'Fun' area but below the horizontal axis due to having electrical output from a power storage unit. The Playpump, referred to in Fig. 5, is another interesting design example, although this design is not powered by electricity, comprising two functions from one application of physical kinetic energy. The purpose of this design is to provide a clean drinking water facility. When children have fun spinning on the PlayPump merry-go-round, clean water is being pumped from underground into a 2,500-litre tank which stands seven meters above the ground [5]. The Playpump will be mapped within the zone of 'fun interaction' but will be place above horizontal axis due to this system not amplifying any electrical output.

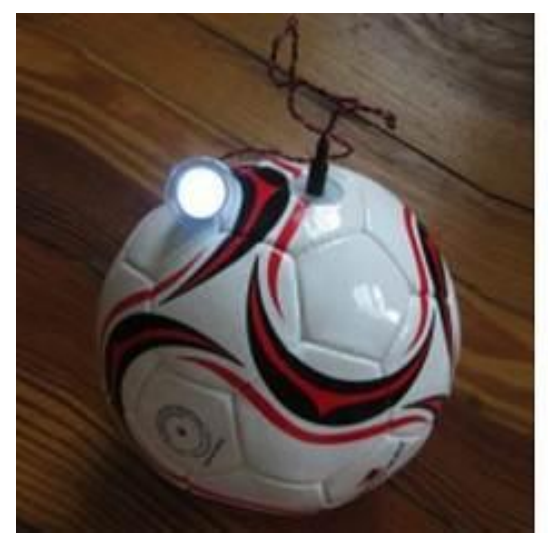

Fig. 4. Soccket [4]

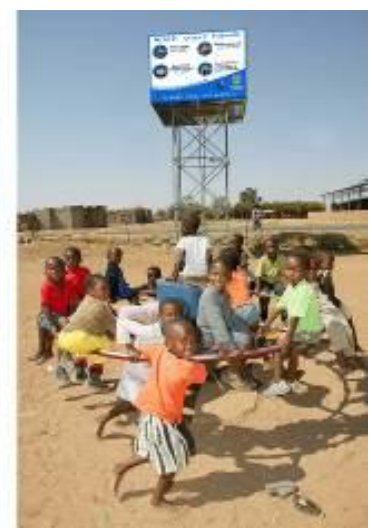

Fig. 5. Playpump [5]

Attributes. This research identifies 5 types of attributes after reviewing the description of interactions and mechanisms of HPP. They are as follows:

- Mechanical + Sub-conscious interaction - Domain A

- Mechanical + Conscious interaction - Domain B

- Electrical + Sub-conscious interaction - Domain D

- Electrical + Conscious interaction - Domain C

- Fun Interaction - Domain F

\section{Case study - 'Off the grid' project}

A case study was conducted with first year product design undergraduate students at Nottingham Trent University. The project addressed the problem of over-energy consumption from the use of everyday electronics. Students were briefed to select an electronic product and (re)design it into an interactive 'off the grid product', meaning its functional power is not being supplied by either the power grid nor any kind of technology driven power units such as photovoltaic power cells. The tasks 
set in the project aimed to direct students to perform a thorough observation on a particular product, helping them to realise how a designed product is specified against a range of complex parameters other than simply performance and aesthetics. Students were required to define, through observation and interrogation, a clear understanding of product functionality, technological requirements, engineering, power systems, manufacturing, and so on. This enabled students to critically explore a single product, approaching the potential design transformation and embodiment in more diverse and conceptual way. The result of the project produced number of speculative concepts that are design driven, proposed as alternative solutions.

Probe. Another important objective of the project was to collect quantitative data of identifying the range of the most common electronics products used in a students' daily life. Therefore, the project was designed into two parts. The first part of the project was a one day observation, producing an observational sheet comprising images of 25 products that they interact the most in their daily routine. All the product items submitted on the observation sheets were then analysed. $44 \%$ of all items were electronic, of which $35 \%$ were classified as 'portable' electronic devices which potentially have a higher possibility of transformation into HPP. The second stage of research classified these products in order to produce further concepts and new artefacts. The project design strategy which incorporates a method of retrieving common items/products data from this type of probe exercise seems a justifiable method to select such products for consideration of (re)designing into HPP. It was deemed crucial as a research objective to deploy methodologies to select and conceptualise such products into HPP which can ideally be acceptable to future users; in some cases enabling the potential creation of new market scenarios. In other words, they must be products that are commonly being used, and will be used to great effect on future products. More detailed methodologies are planned to utilise these results to develop appropriate theoretical and tangible artefacts to support this hypothesis.

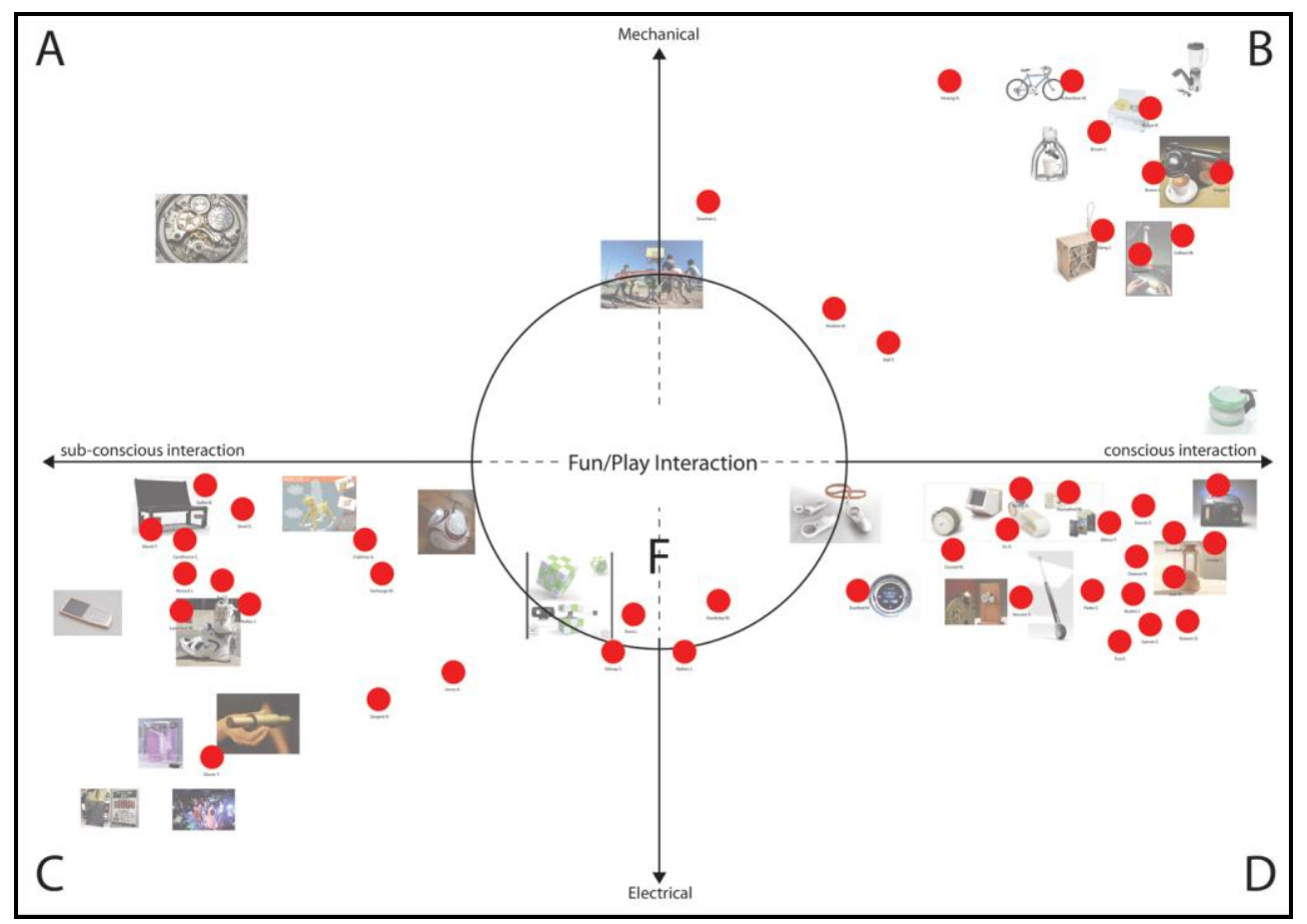

Fig. 6. Overlay mapping (Red dot shows the mapping of the student project)

Mapping. After the first part of the project, students were briefed to select an electronic product from their observational sheet and (re)design it into an interactive 'off the grid' product. All of the students' final concepts were characterised and mapped onto the 'Interaction Map'. It produced a 
comparative analysis with the current research result from monitoring both tangible (real) products and concepts of HPP. Figure 6 shows the overlay mapping of real-time HPP concepts and the mapping result of the student project. It can be observed that the results from both cases showed similar mapping characteristics The HPP monitoring process has only found one example that best describes the domain A which was a Pendulum Watch [6]. However, the case study exercise was not able to identify any concepts or innovative idea arising from students' concepts that could be characterised and mapped within domain A.

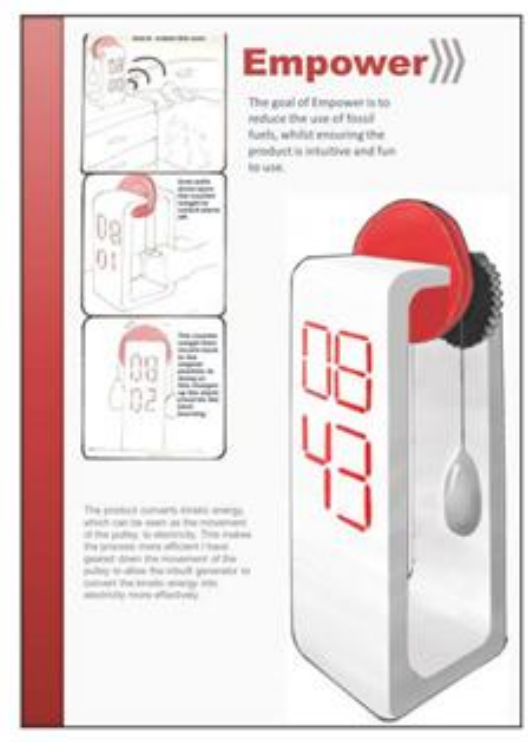

Fig. 6. Empower

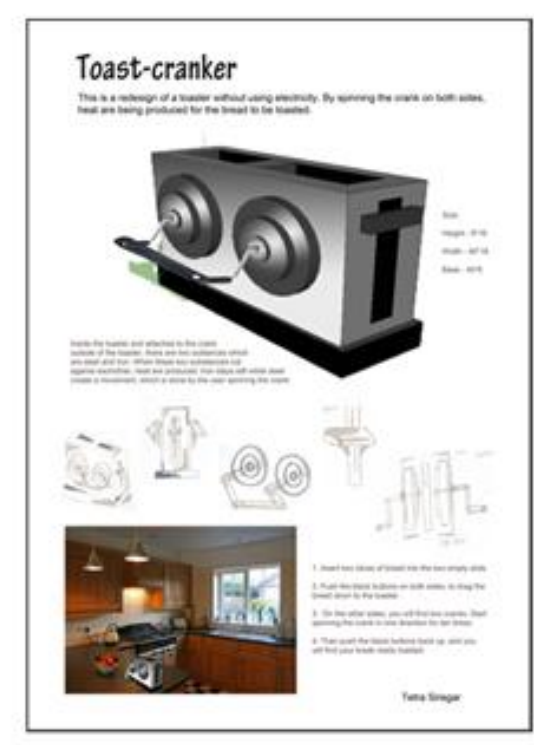

Fig. 7. Toast-Cranker

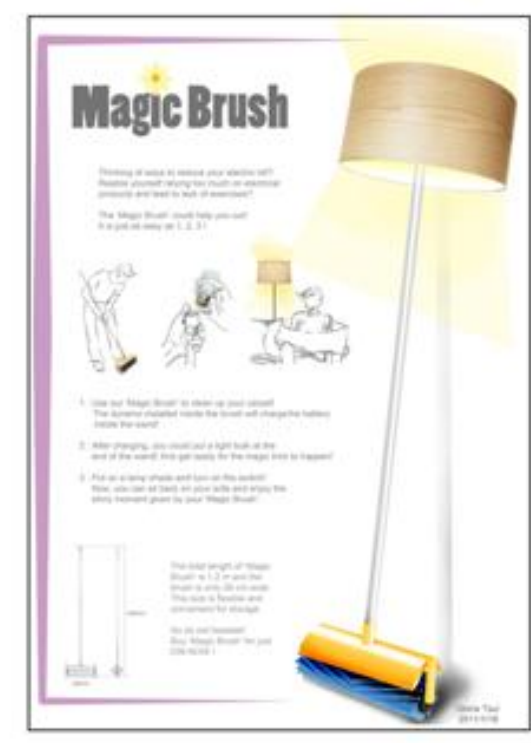

Fig 8. Magic Brush

Concepts. Above figures show 3 speculative concepts resulted from this case study, produced by student, proposing as an alternative to solution.

- Figure 6, Empower (Domain D): It is a human-powered clock that generates electricity when the user pulls down upon the counter weight to switch the alarm off. The counter weight then recoils back to the original position. In doing so, this charges up the alarm clock for the next morning. It gears down the movement of the pulley to allow the inbuilt generator to convert the kinetic energy into electricity.

- Figure 7, Toast-Cranker (Domain B): Inside the toaster and attached to the crank (which is rotated on the outside of the toaster), there are two metal substrates or plates When these two substrates rub against each other, heat is produced, and therefore the potential to create toast..

- Figure 8, Magic Brush (Domain D): A roller brush, sweeping the carpet floor, is attached to a wand which contains a battery.. A dynamo is embedded inside the roller brush which allows the user to generate electricity as they sweep. A replaceable LED bulb and shade are attached on the top of the wand, expressed as a secondary function as a standing light. The user can detach then bulb if required when sweeping, and simply re-attach when used as a mobile standing light.

\section{Conclusion}

The focus of the research to date is the investigation of the role of designer in the field of human-powered product development. It also aims to investigate empirically proved benefits of HPPs, yet defined, that can be applied from a design perspective. Initially, it is important to 
understand the characteristics of current HPP streams of activity. Therefore, the 'Interaction Map' can be used as a framework for analysing concepts; and as a tool to forecast the potential design spaces, future scenarios/ideas of human-powered products. It is expected that in the near future, all of the identified attributes will be further examined and revised into more concise and distinctive definitions.

Also, the HPP concepts presented in this paper highlight the need for technologies that enable human power conversion into functional power for such device or concept. Integrating knowledge of both current and future electrical and mechanical technologies is integral to the development of concepts and scenarios. Therefore, the design approach undertaken in the case study highlights potential applications for human-power systems, and insight into future technological opportunities for HPP. Beyond the current state of over-reliance on alternative or traditional power sources, the research attempts to create further persuasive arguments that there are other notions to consider in a wider design context.

\section{References}

[1] T.T. Baylis, Baylis Generators Limited (Twickenham, GB), United States Patent 5,917,310, 1999. [2] O. Yousuke, 2008, Revamped 'Power Generating Floor' to Be Tested at Tokyo Station, [online] Available at: http://techon.nikkeibp.co.jp/english/NEWS_EN/20081204/162357/, [Accessed 03-26 2011]

[3] A. Jansen, Industrial Design Enginering, Delft University of Technology, PhD Thesis, Delft, 2011.

[4] Soccket, [online] Available at: http://www.soccket.com/, [Accessed 15-03 2011]

[5] W.f. People, Playpump, [online] Available at:

http://www.waterforpeople.org/extras/playpumps/case-foundation-partnership.html, [Accessed 11-10 2010]

[6] T. Heuer, TAG HEUER PENDULUM [online] Available at:

http://www.tagheuer.com/the-collection/concept-watches/pendulum-concept/index.lbl?lang=en, [Accessed 20-04 2011] 